\title{
Computational and Experimental Evidence for the Structural Preference of Phenolic C-8 Purine Adducts
}

Andrea L. Millen, ${ }^{\dagger}$ Christopher K. McLaughlin, ${ }^{\dagger}$ Kewen M. Sun, ${ }^{\ddagger}$ Richard A. Manderville ${ }^{\star *}$ and Stacey D. Wetmore ${ }^{\dagger, *}$

Department of Chemistry, University of Lethbridge, 4401 University Drive, Lethbridge, Alberta, Canada, T1K 3M4, and the Department of Chemistry, University of Guelph, Guelph, Ontario, Canada, N1G 2W1

\section{Supporting Information}

Figures S1-S8, and Tables S1 and S2 as described in the text.

${ }^{\dagger}$ University of Lethbridge.

University of Guelph.

* To whom correspondence should be addressed. E-mail: stacey.wetmore@uleth.ca, and rmanderv@uoguelph.ca. 


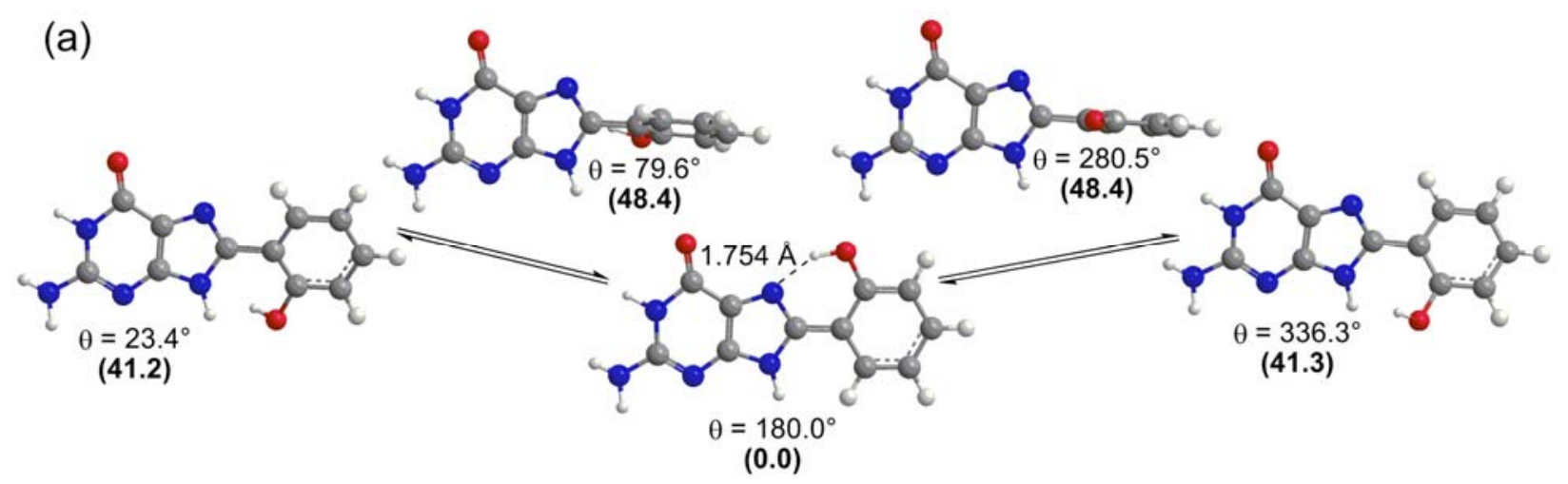

(b)

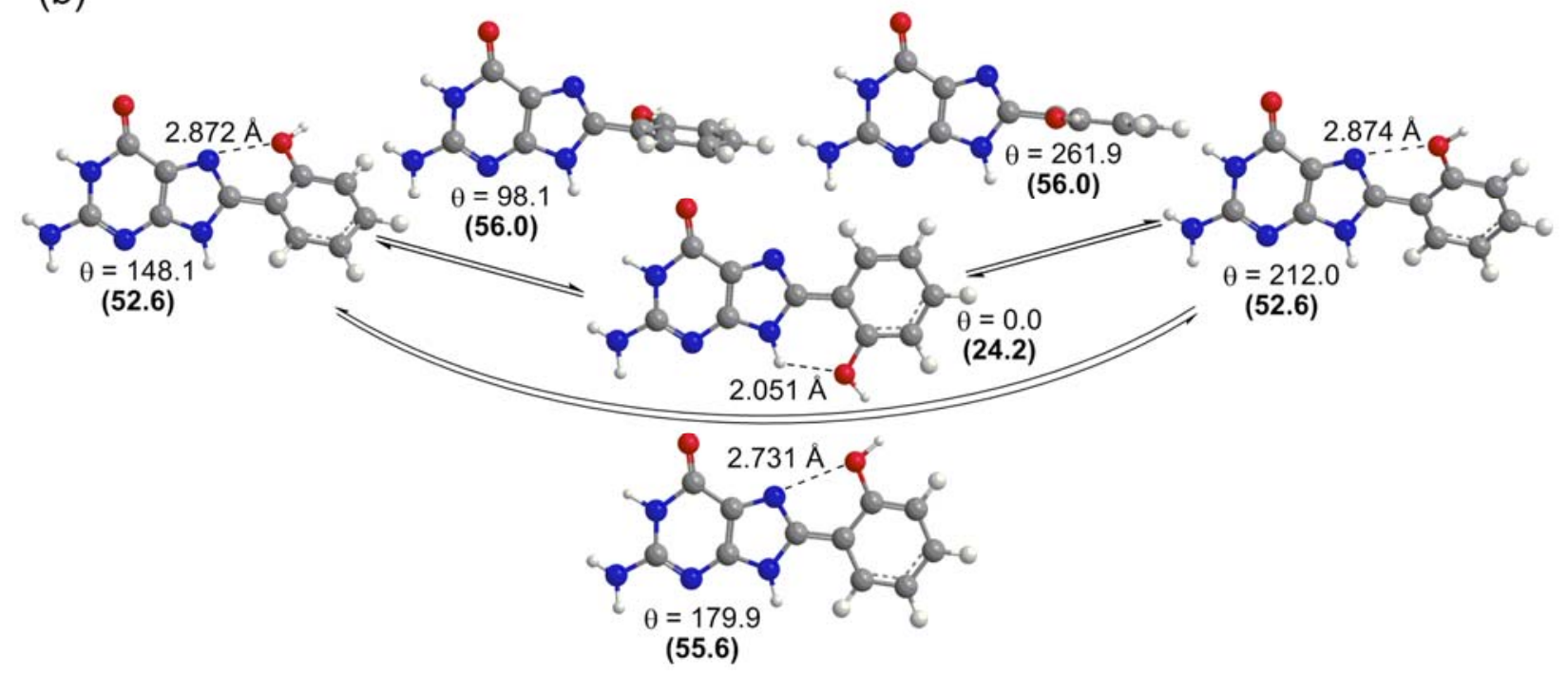

Figure S1. Selected B3LYP/6-31G(d) bond lengths $(\AA)$ and angles $(\theta$, deg.,) of deglyco1 with a) $\angle\left(\mathrm{HOC}_{11} \mathrm{C}_{10}\right) \approx 0^{\circ}$ and $\left.\mathrm{b}\right) \angle\left(\mathrm{HOC}_{11} \mathrm{C}_{10}\right) \approx 180^{\circ}(\mathrm{B} 3 \mathrm{LYP} / 6-311+\mathrm{G}(2 \mathrm{df}, \mathrm{p})$ relative energies from single-point calculations provided in parenthesis, $\left.\mathrm{kJ} \mathrm{mol}^{-1}\right)$. 


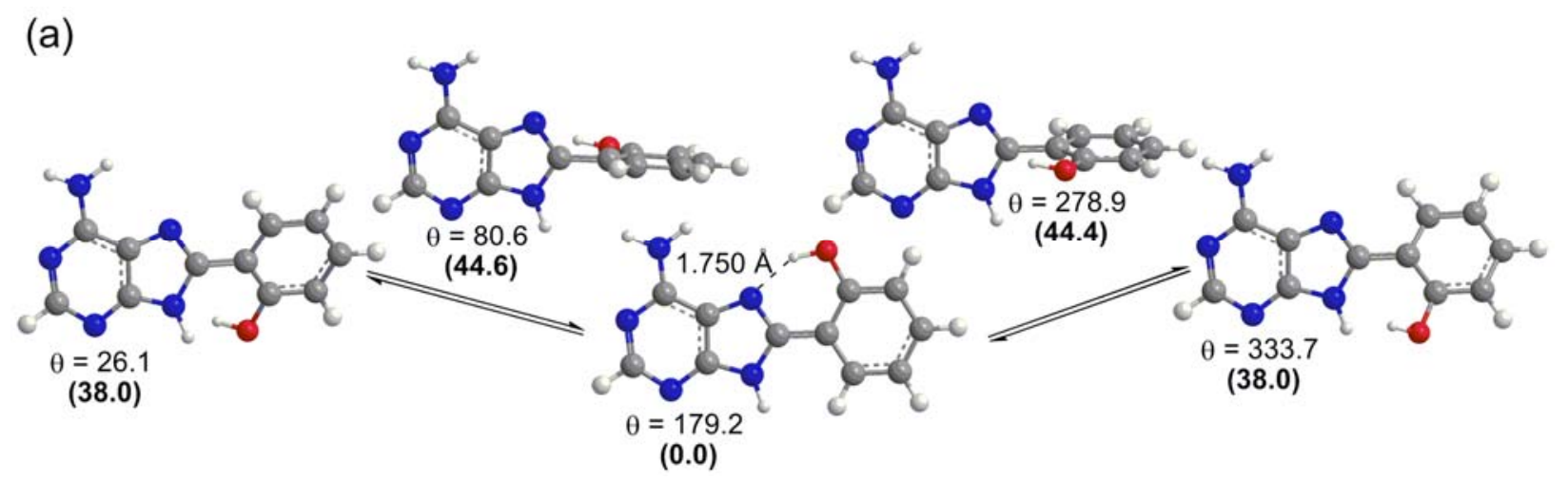

(b)

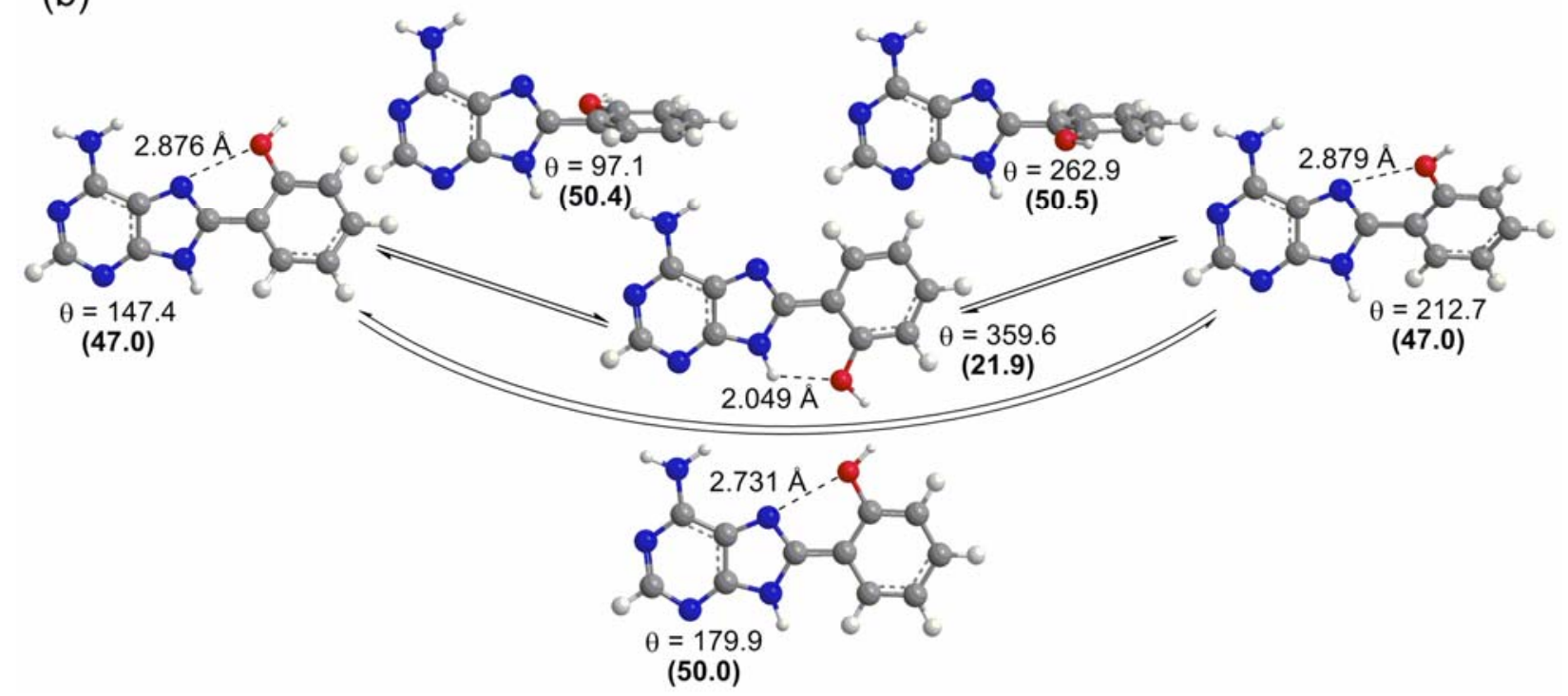

Figure S2. Selected B3LYP/6-31G(d) bond lengths $(\AA)$ and angles $(\theta$, deg., $)$ of deglyco2 with a) $\angle\left(\mathrm{HOC}_{11} \mathrm{C}_{10}\right) \approx 0^{\circ}$ and $\left.\mathrm{b}\right) \angle\left(\mathrm{HOC}_{11} \mathrm{C}_{10}\right) \approx 180^{\circ}(\mathrm{B} 3 \mathrm{LYP} / 6-311+\mathrm{G}(2 \mathrm{df}, \mathrm{p})$ relative energies from single-point calculations provided in parenthesis, $\left.\mathrm{kJ} \mathrm{mol}^{-1}\right)$. 


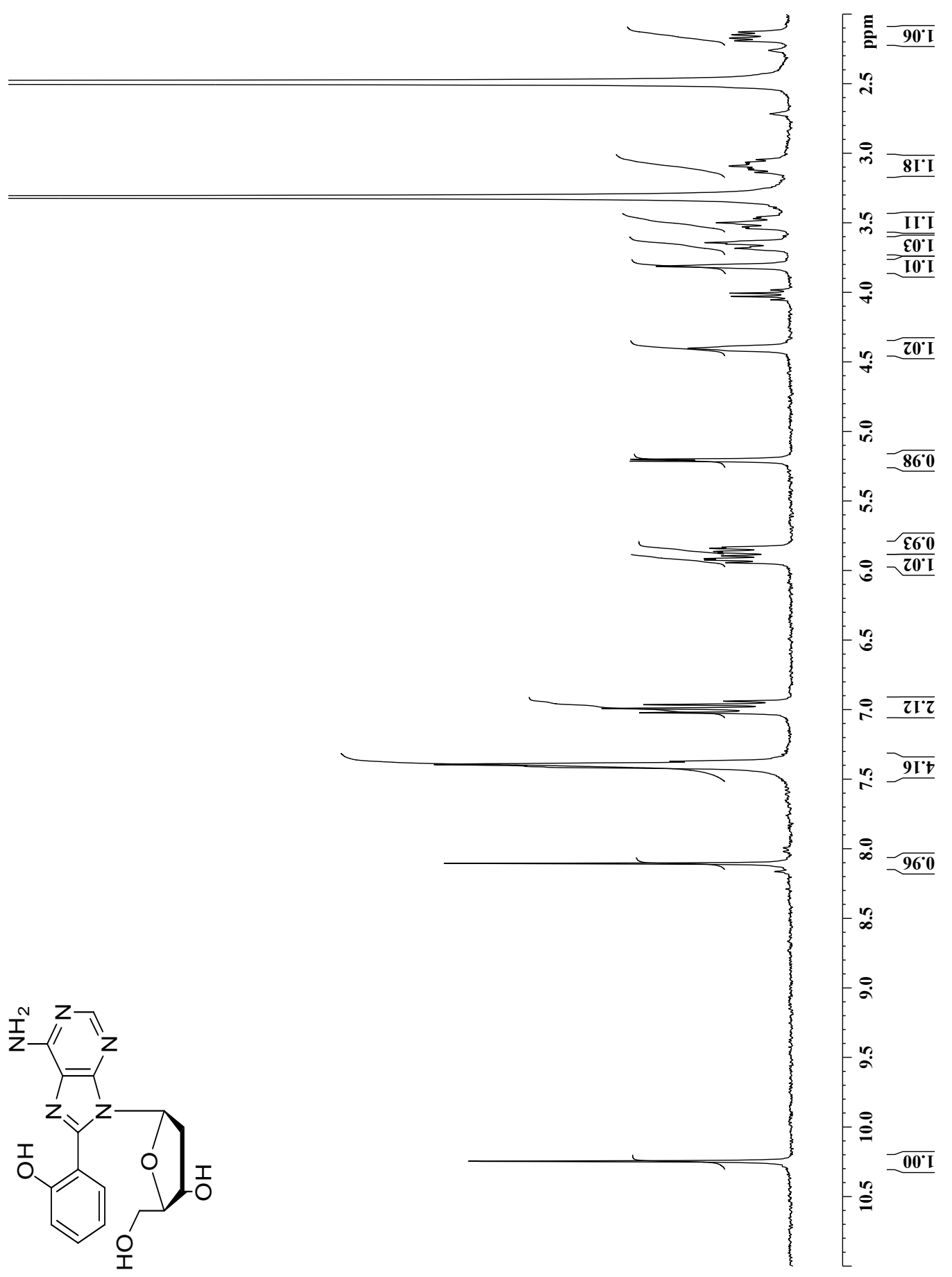

Figure S3. ${ }^{1} \mathrm{H}$ NMR spectra of 2 in DMSO-d ${ }_{6}$. 


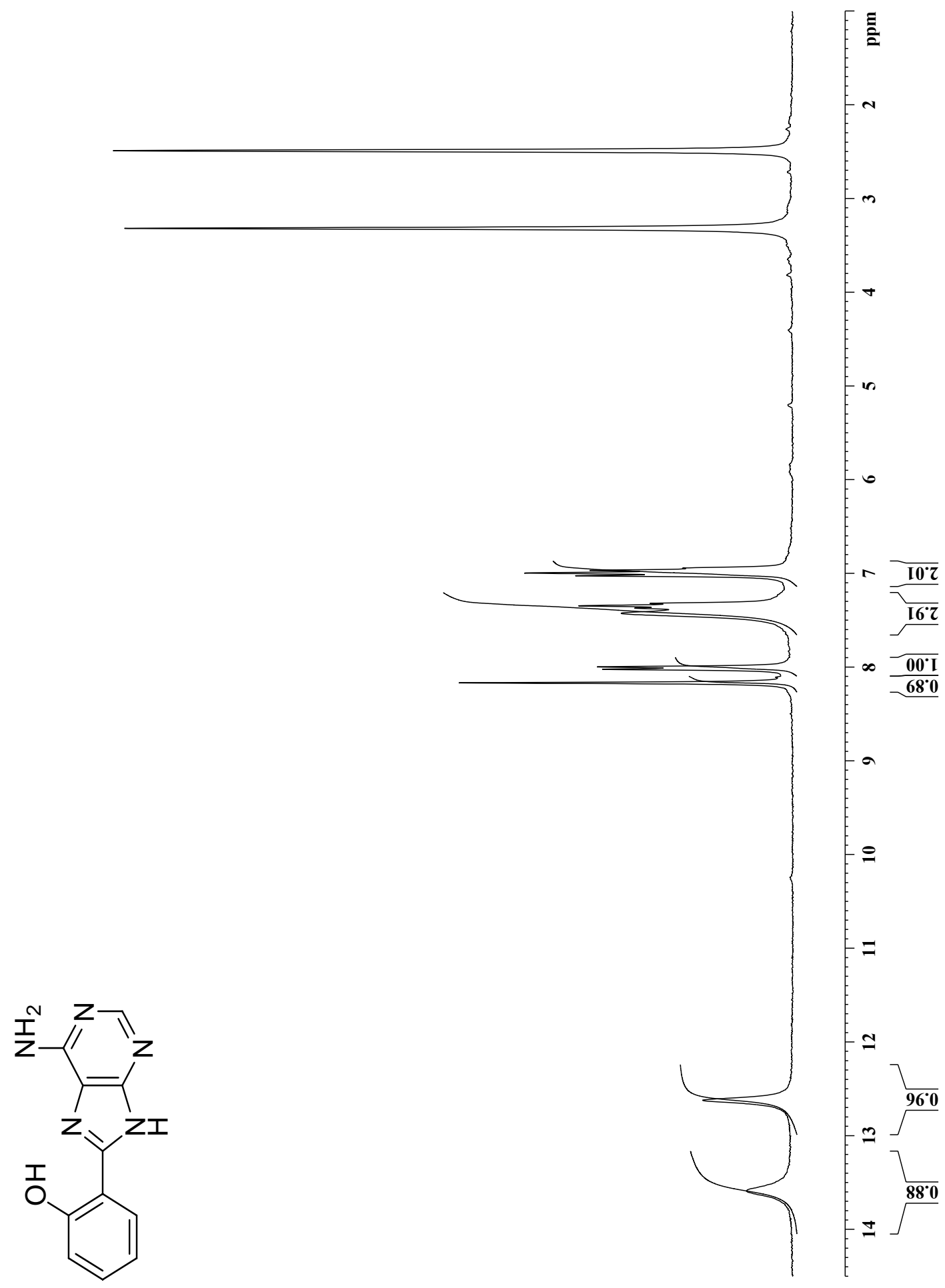

Figure S4. ${ }^{1} \mathrm{H}$ NMR spectra of deglyco2 in DMSO-d ${ }_{6}$. 


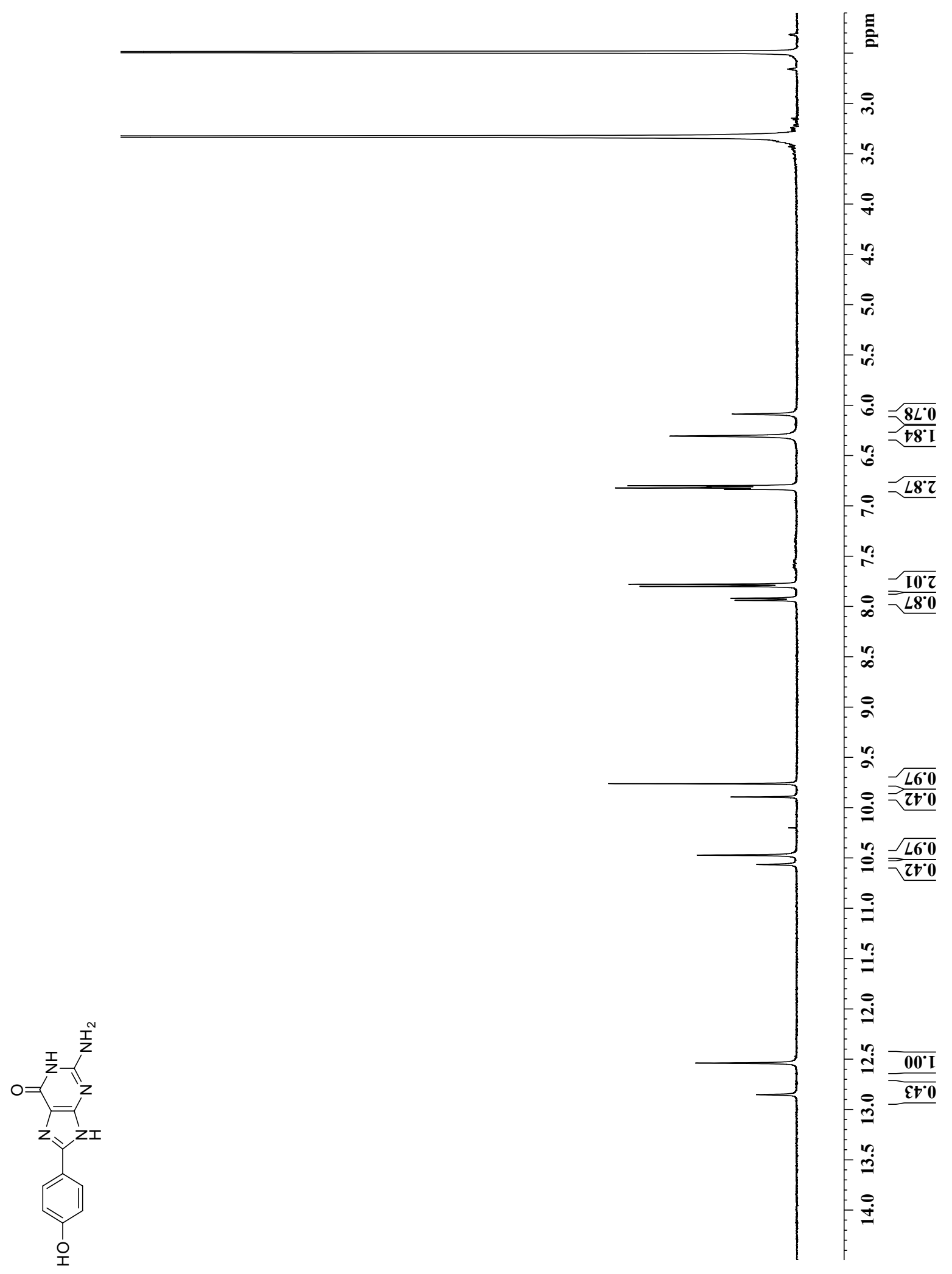

Figure S5. ${ }^{1} \mathrm{H}$ NMR spectra of deglyco3 in DMSO-d ${ }_{6}$. 


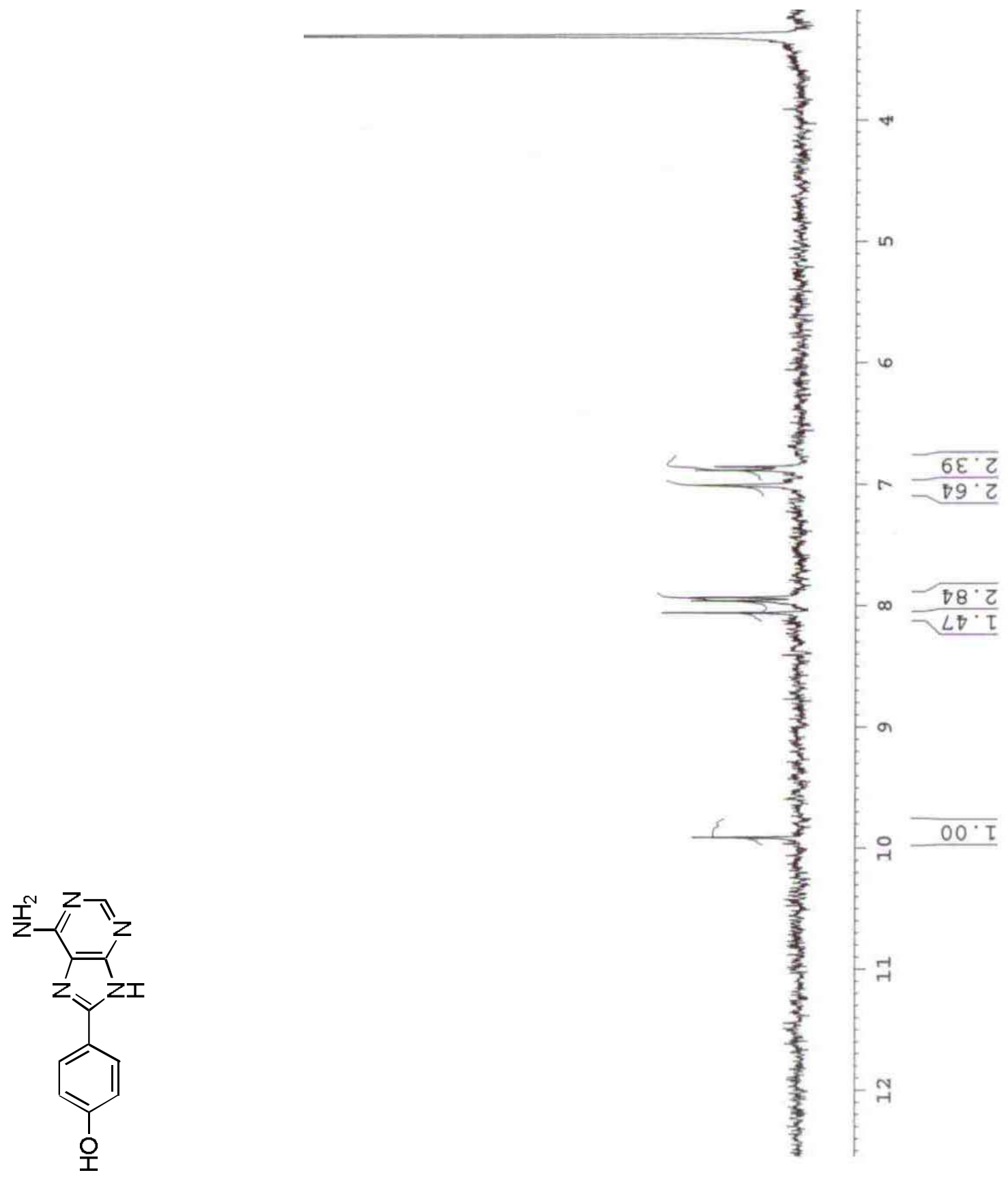

Figure S6. ${ }^{1} \mathrm{H}$ NMR spectra of deglyco4 in DMSO-d 6 . 


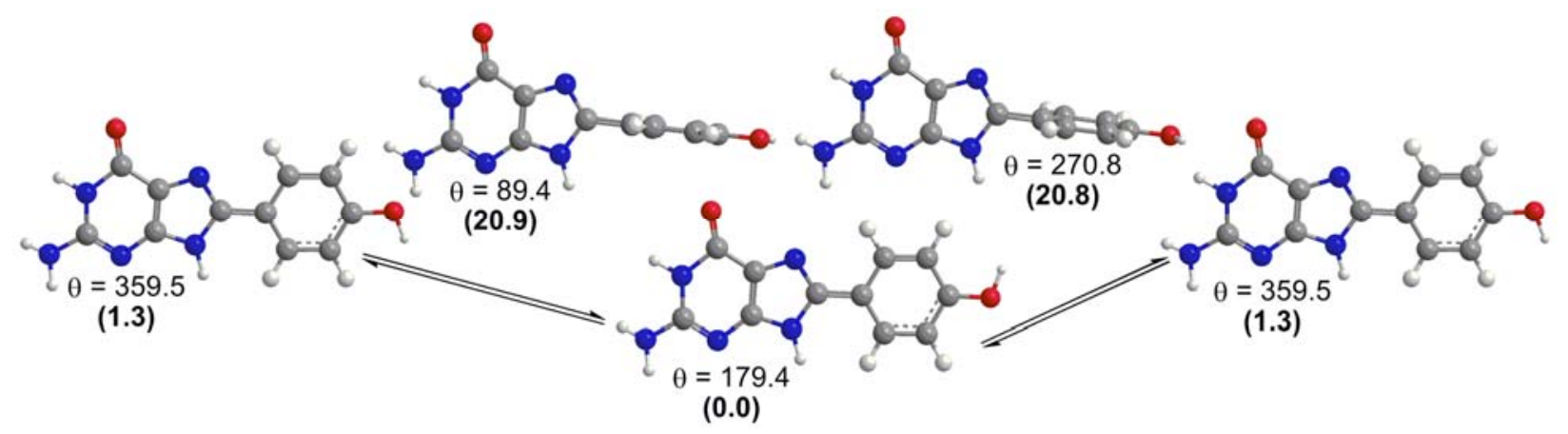

Figure S7. Selected B3LYP/6-31G(d) bond lengths $(\AA)$ and angles ( $\theta$, deg.,) of deglyco3 (B3LYP/6-311+G(2df,p) relative energies from single-point calculations provided in parenthesis, $\left.\mathrm{kJ} \mathrm{mol}^{-1}\right)$.

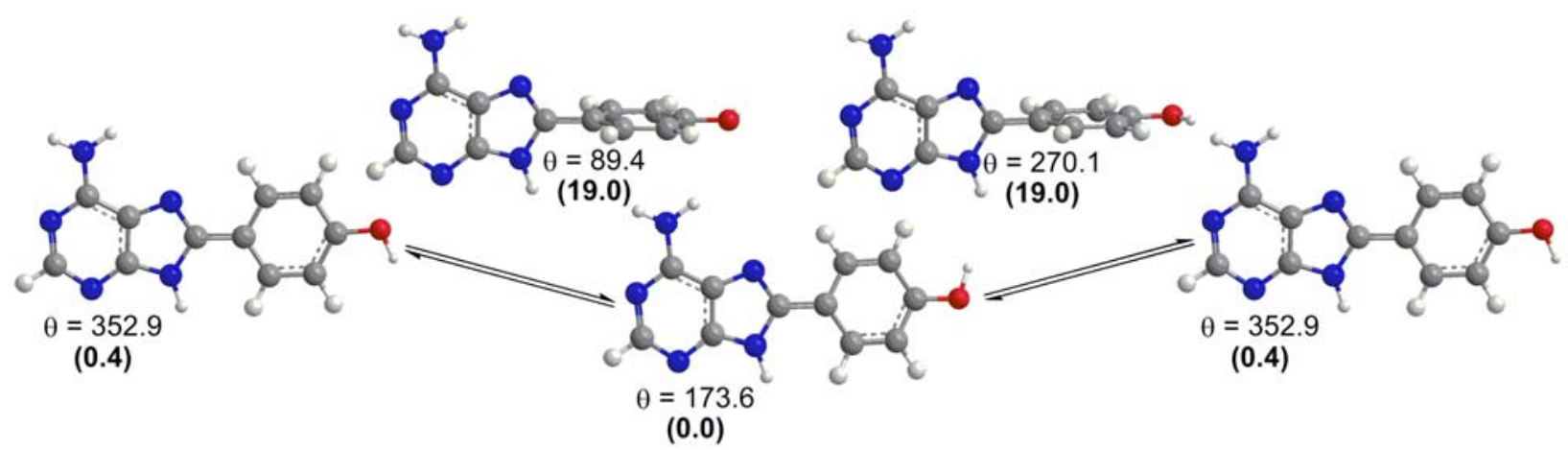

Figure S8. Selected B3LYP/6-31G(d) bond lengths $(\AA)$ and angles ( $\theta$, deg.,) of deglyco4 $(\mathrm{B} 3 \mathrm{LYP} / 6-311+\mathrm{G}(2 \mathrm{df}, \mathrm{p})$ relative energies from single-point calculations provided in parenthesis, $\left.\mathrm{kJ} \mathrm{mol}{ }^{-1}\right)$. 
Table S1. Geometrical Data and Relative Energies of Syn Minima and Transition States for 1 and 2 Optimized in Various Solvents ( $\chi$ and $\theta$ in deg., relative energies in $\mathrm{kJ} \mathrm{mol}^{-1}$ )

\begin{tabular}{|c|c|c|c|c|c|c|c|c|c|c|c|c|}
\hline & \multicolumn{2}{|c|}{ Gas Phase } & \multicolumn{2}{|c|}{ Cyclohexane } & \multicolumn{2}{|c|}{ Chloroform } & \multicolumn{2}{|c|}{ Acetonitrile } & \multicolumn{2}{|c|}{ DMSO } & \multicolumn{2}{|c|}{ Water } \\
\hline & $\mathbf{A}$ & $\mathbf{G}$ & A & $\mathbf{G}$ & A & $\mathbf{G}$ & $\mathbf{A}$ & $\mathbf{G}$ & $\mathbf{A}$ & $\mathbf{G}$ & $\mathbf{A}$ & $\mathbf{G}$ \\
\hline$\chi$ & 71.0 & 66.2 & 71.1 & 66.5 & 71.3 & 66.5 & 71.3 & 63.1 & 71.7 & 66.1 & 75.0 & \\
\hline$\tilde{\theta}$ & 53.7 & 53.4 & 54.0 & 54.2 & 54.6 & 54.2 & 55.0 & 57.3 & 55.4 & 55.8 & 58.6 & \\
\hline$\Delta \mathbf{E}$ & 19.1 & 24.4 & 18.3 & 22.9 & 19.2 & 20.9 & 19.4 & 18.1 & 19.3 & 19.5 & 11.4 & \\
\hline$\chi$ & 56.1 & 55.1 & 56.3 & 55.2 & 56.3 & 55.3 & 56.3 & 64.2 & & 55.4 & & 52.4 \\
\hline$\tilde{\theta}$ & 87.4 & 85.0 & 87.0 & 85.4 & 86.8 & 85.0 & 85.0 & 54.1 & & 84.4 & & 87.9 \\
\hline$\Delta \mathbf{E}$ & 20.4 & 25.0 & 19.2 & 23.0 & 17.9 & 19.8 & 16.2 & 26.3 & & 17.8 & & 11.0 \\
\hline$\chi$ & 43.8 & 44.1 & 44.0 & 44.3 & 44.0 & 44.6 & 44.1 & 44.6 & 44.2 & 44.7 & 41.1 & 41.9 \\
\hline$\theta$ & 153.8 & 156.7 & 153.5 & 155.3 & 153.2 & 155.0 & 153.0 & 154.3 & 152.9 & 154.2 & 152.8 & 153.0 \\
\hline$\Delta \mathbf{E}$ & 0.3 & 0.5 & 0.0 & 0.3 & 0.0 & 0.2 & 0.0 & 0.2 & 0.0 & 0.0 & 0.0 & 0.0 \\
\hline$\chi$ & 53.7 & 53.5 & 53.5 & 53.5 & 53.4 & 53.7 & 53.2 & 53.5 & 53.4 & 53.7 & 48.5 & 48.8 \\
\hline $\boldsymbol{\theta}$ & 185.0 & 185.7 & 185.2 & 185.6 & 185.3 & 185.5 & 185.5 & 185.7 & 185.5 & 185.6 & 190.1 & 189.9 \\
\hline$\Delta \mathbf{E}$ & 12.9 & 11.2 & 12.9 & 11.3 & 13.4 & 10.9 & 13.8 & 12.3 & 14.0 & 11.3 & 16.8 & 16.0 \\
\hline$\chi$ & 73.2 & 71.0 & 73.0 & 71.4 & 72.8 & 71.9 & 72.8 & 72.6 & 72.6 & 72.4 & 73.4 & 71.8 \\
\hline $\boldsymbol{\theta}$ & 208.0 & 207.0 & 208.1 & 206.9 & 208.0 & 206.8 & 207.8 & 206.8 & 207.8 & 206.7 & 208.5 & 209.1 \\
\hline$\Delta \mathbf{E}$ & 0.0 & 0.0 & 0.0 & 0.0 & 0.7 & 0.0 & 1.1 & 0.0 & 1.3 & 0.0 & 4.2 & 4.4 \\
\hline$\chi$ & 53.4 & 53.3 & 53.6 & 53.3 & 54.0 & 53.5 & 54.6 & 53.9 & 54.5 & 53.8 & & \\
\hline$\theta$ & 276.4 & 277.5 & 275.4 & 276.9 & 274.1 & 275.3 & 272.3 & 274.6 & 272.1 & 273.5 & & \\
\hline$\Delta \mathbf{E}$ & 21.1 & 24.8 & 19.8 & 22.9 & 18.7 & 20.3 & 17.4 & 18.1 & 17.3 & 17.8 & & \\
\hline$\chi$ & 47.7 & 48.0 & 48.1 & 48.8 & 49.2 & 49.6 & 49.6 & 50.3 & 49.3 & 50.4 & 45.3 & 48.8 \\
\hline$\tilde{\theta}$ & 304.8 & 304.4 & 304.2 & 302.8 & 302.0 & 301.1 & 302.3 & 299.8 & 302.4 & 299.7 & 301.2 & 296.4 \\
\hline$\Delta \mathbf{E}$ & 22.1 & 26.6 & 21.0 & 24.9 & 19.7 & 21.9 & 18.2 & 20.2 & 18.8 & 19.8 & 5.9 & 6.2 \\
\hline
\end{tabular}

Table S2. Absorption data for 1, 2, deglyco1 and deglyco2

\begin{tabular}{llll}
\hline Adduct & $\lambda_{\max }, \varepsilon_{\text {MOPS }}{ }^{2}$ & $\lambda_{\max }, \varepsilon_{\text {DMSO }}$ & $\lambda_{\max }, \varepsilon_{\text {MeCN }}$ \\
\hline $\mathbf{1}$ & 276,17932 & 282,17814 & 281 and 318 \\
$\mathbf{2}$ & 270,17036 & 283,16924 & 279 and 315 \\
deglyco1 & 319,18887 & 327,20874 & 322,21349 \\
deglyco2 & 317,14369 & 325,15944 & 323,16307 \\
\hline
\end{tabular}

${ }^{\mathrm{a}}$ MOPS buffer $(10 \mathrm{mM}) \mathrm{pH} 7.0$ containing $0.1 \mathrm{M} \mathrm{NaCl}$. 